\title{
An Experimental Momentum-based Front Detection Method for Autonomous Underwater Vehicles
}

\author{
Jeremy Gottlieb, Rishi Graham, Thom Maughan, Frédéric Py, \\ Gabriel Elkaim and Kanna Rajan
}

\begin{abstract}
Fronts have been recognized as hotspots of intense biological activity and are important targets for observation to understand coastal ecology and transport in a changing ocean. With high spatial and temporal variability, detection and event response for frontal zones is challenging for robotic platforms like autonomous underwater vehicles (AUVs). These vehicles have shown their versatility and cost-effectiveness in using automated approaches to detect a range of features. Targeting them for in-situ observation and sampling capabilities for frontal zones then provides an important tool for characterizing rapid and episodic changes. We introduce a novel momentumbased front detection (MBFD) algorithm which utilizes a Kalman filter and a momentum accumulator function to identify significant temperature gradients associated with upwelling fronts. MBFD is designed to work at a number of levels including onboard an AUV, on-shore with a sparse real-time data stream and post-experiment on a full resolution data set gathered by a vehicle. Such a multi-layered approach plays an important role in mixed human-robot decision making for oceanographers making coordinated sampling and asset allocation strategies in large multi-robot field experiments in the coastal ocean.
\end{abstract}

\section{INTRODUCTION AND MOTIVATION}

The coastal ocean is a complex environment driven by the interaction of atmospheric, oceanographic, estuarine/riverine and land-sea processes. Among the phenomena that oceanographers study are ocean fronts - zones of locally intensive physical gradients that experience enhanced circulation and biological activity. Fronts are important targets for observation to understand coastal ecology and transport. They occur at varying spatial scales, from a depth of a few meters all the way to the sub-sea benthic environment. They also occur at highly variable temporal scales, subsisting from a few hours to a few weeks. In addition, they can be observed via changes in multiple environmental properties (e.g., temperature, nitrate, salinity, etc).

Studying fronts has been a persistent challenge. Recently, approaches using robotic vehicles have been attempted but recognition of the frontal boundary remains difficult. Thus far, a reliable mathematical model of the structure and behavior of fronts does not exist. As a consequence, computational methods and those tied to autonomic behavior are sparse. Instead, oceanographers

J. Gottlieb and G. Elkaim are with the Baskin School of Engineering, UC Santa Cruz, 1156 High St., Santa Cruz, CA 95064 USA $\{$ gottlieb, elkaim\}asoe.ucsc.edu

R. Graham, T. Maughan, F. Py, and K. Rajan are with MBARI, 7700 Sandholdt Rd., Moss Landing, CA 95039 USA \{rishi, tm, fpy, kanna.rajan\}@mbari.org

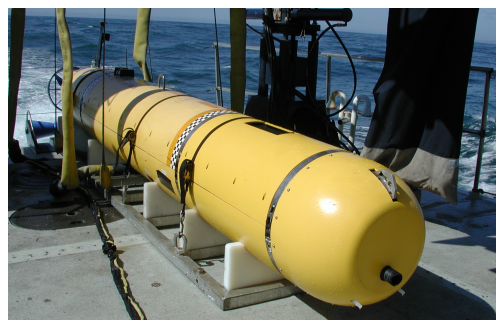

Fig. 1: MBARI's Dorado AUV on the R/V Zephyr.

use heuristics based on ship-board and remote sensing measurements to characterize frontal zones.

Autonomous underwater vehicles (AUVs) in this context offer new, more cost-effective and sustainable opportunities for making targeted oceanographic observations at high spatial and temporal resolution. AUVs with the ability to automatically detect features can autonomously retarget themselves to detect and then track fronts, potentially making water sample measurements of interest to oceanographers[12]. Often scientists are interested in sampling the differing water mass across and within the frontal boundaries. This is difficult enough for scientists on manned vessels. Our end goal is to have a robot undertake such sampling missions autonomously. The primary challenges include translating the heuristics that oceanographers use to identify fronts into features a robot can use for identification, and identifying these features in the context of a very noisy environmental background.

We introduce a novel momentum-based front detection (MBFD) algorithm designed for use on AUVs. The algorithm has been run onboard MBARI's Dorado platform (Fig. 1). Simultaneously, the algorithm is also run on shore within the Oceanographic Decision Support System (ODSS) [6] for the Controlled, Agile, and Novel Observing Network (CANON) program [1]. It is an early attempt to implement event response capabilities in the coastal ocean, a key scientific and engineering goal for CANON and the ODSS. Dynamic and episodic phenomena such as fronts are hard to predict given current difficulties constructing ocean models. Thus, having robotic platforms that can autonomously and dynamically target these phenomena would be beneficial to oceanographers. We envision future applications that would allow an AUV to use these algorithms to react to the presence of fronts in order to clarify their location or direction of movement. While the technique presented 


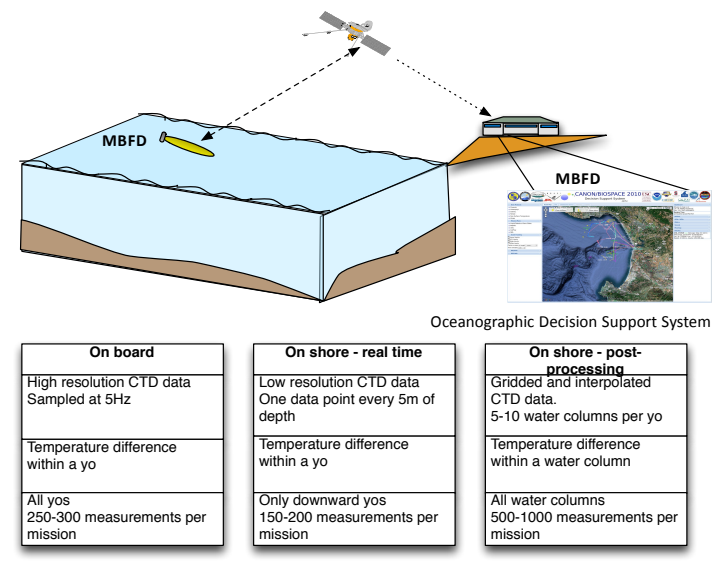

Fig. 2: The experimental setup for running MBFD onboard the AUV, using sub-sampled AUV data obtained via satellite and using full-resolution log data.

can be applied to any associated oceanographic property, our discussion will focus primarily on temperature.

Our work extends earlier methods by applying statistical parameter estimation from past data characterizing the extent of the transition zone that defines an ocean front. The experimental setup is as shown in Fig. 2. MBFD is run in three contexts: onboard the Dorado AUV; on sub-sampled AUV data sent to shore via satellite; and post-hoc after mission completion on full resolution data. This work was specifically targeted at identifying upwelling fronts.

The paper is organized as follows: Section II describes related work. Section III describes the algorithm while Section IV highlights the results of MBFD on archived data as well as on an AUV deployment at sea. Section V analyses the results and Section VI concludes with future work.

\section{RELATED WORK}

Fronts have been studied primarily by physical oceanographers applying edge detection techniques to remote sensing profiles of sea-surface temperature (SST) measurements ([14], [9], [11], [3]) where [4] forms the basis for many of the detection methods. Biological productivity in fronts has been a long-term study topic (e.g., [15]), while the impact of fronts on large marine ecosystems are an important factor in the study of coastal ecosystem management [8], [13], [10].

Traditional ship based observations such as recent fine scale measurements using towed instruments have been the norm in the ocean sciences [7]. Automated approaches are relatively new; newer still are those used in-situ on marine robots, particularly those using a fullvolume field (horizontal and vertical measurements). The primary advantage to using AUVs over ship-based observations is that AUVs can be in the water continuously for longer periods cost-effectively. Unpowered AUVs or gliders can persist for months in the watercolumn driven by human operators via satellite.
[5] used AUVs to reactively detect and track a thermocline. While their method as applied to the thermocline is similar in appearance to ours as applied to fronts, they seek to identify a specific point as the centroid of the region of intense change, while we seek to define the entire region over which change is intense. Earlier work from [16] made use of mixed-initiative methods to target fronts; field reconstruction from sub-sampled data is used by an oceanographer on shore for identifying a front, followed by retargeting of the vehicle.

An upwelling front can be characterized by a rapid transition between stratified and non-stratified water. By definition, stratified water contains a strong vertical gradient. In [17], vertical gradient information is captured by computing the temperature difference between two predefined depths. This method is extended in [18] to quantify the horizontal gradient of the vertical temperature difference as a basis for front detection. These algorithms have been demonstrated on AUVs in situ for autonomous detection of stratified and unstratified water types as well as the fronts between them. Our work follows this approach using a Kalman filter to smooth the signal and a momentum function to aggregate change over distance.

\section{The MBFD ALgorithm}

MBFD was designed to be flexible and scalable in multiple data-gathering contexts, using parameter values estimated from past data. Momentum values are generated directly from the data by taking into account the magnitude of vertical differences and horizontal cumulative change. While [17], [18] use differences at discrete depths, our measure is the maximum difference within a continuous profile.

\section{A. The Role of Gradients in Fronts}

Fig. 3 shows an example of a water-column track with a temperature front encountered by our AUV during an overnight mission on June 13-14, 2011. The AUV was typically targeted at a suspected frontal region by oceanographers using remote sensing and archival data coupled with their understanding of the mechanics of

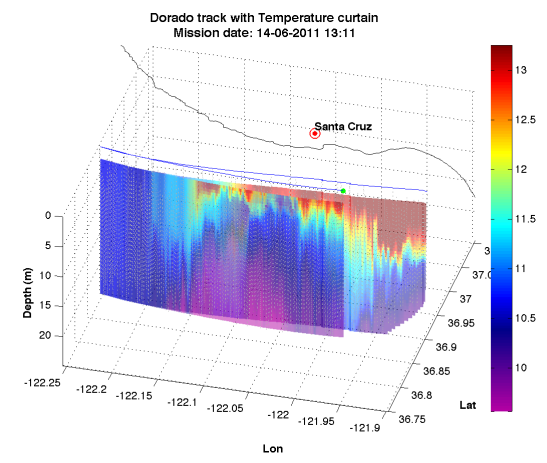

Fig. 3: An AUV track with temperature data showing a frontal zone, from a mission in Monterey Bay in June, 2011. 
upwelling zones in Monterey Bay. However, MBFD itself has no a priori contextual information and is therefore unbiased towards identifying fronts.

Our experimental setup closely follows that of [17], [18]. As the AUV moves through the front into the upwelled water, the temperature within the water column rapidly becomes more homogenous (less as it moves out). The temperature difference decreases (increases), which generates a steep, sustained gradient. In order to characterize the vertical structure of the water column over a region of (horizontal) space, the AUV executes a yo-yo pattern. ${ }^{1}$ The AUV track is then partitioned into a sequence of $N_{\text {prof }} \in \mathbb{N}$ profiles, transects from surface to depth or depth to surface. For each profile, the difference between maximum and minimum measured temperature values is stored.

\section{B. Algorithmic Details}

The maximum vertical difference within the water column is a continuous function of horizontal location. We use the maximum difference across each profile as a discrete proxy, applying a Kalman filter to obtain a smooth approximation of the underlying signal. The resulting approximate value associated with profile $p \in$ $\left\{1, \ldots, N_{\text {prof }}\right\}$ we denote by $\operatorname{mvd}(p)$, and use as a basis for our momentum based accumulation function.

1) The Momentum function: If the correct smoothing parameters were known a priori, then front detection could be a matter of finding the extrema of the instantaneous rate of change of the smoothed signal. However, this approach is too highly variable from one dataset to the next to be practical. Instead, we define an accumulated trend based on the notion of momentum to allow us to quantify a natural trend (Fig. 4). For a given segment of the AUV trajectory, the region over which mvd accumulates the most momentum in a given direction (with

\footnotetext{
${ }^{1}$ The variability we are interested in is in the vertical dimension, but Dorado cannot hover - it must always be moving forward. Hence, the yo-yo pattern is the most efficient mechanism for studying variability across multiple water columns.
}
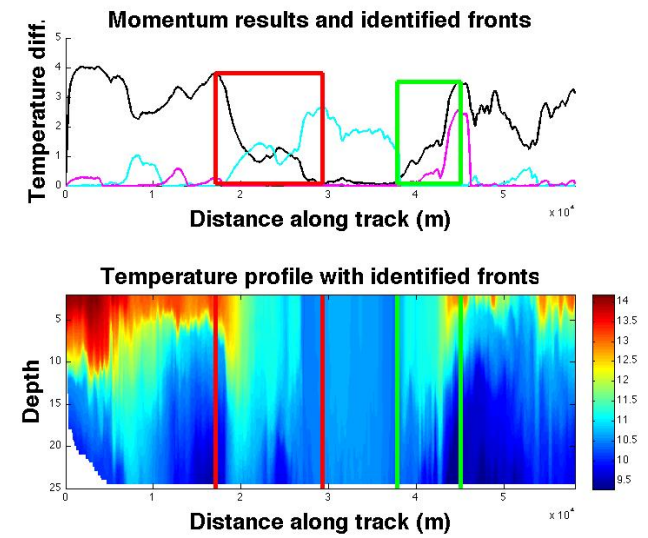

Fig. 4: Temperature data from June 2011, in Monterey Bay. Top plot shows mvd (black), $D M$ (cyan), $U M$ (purple), and identified downward (red box) and upward (green box) fronts. an upward or downward trend) is the best candidate for a front. The downward momentum, $D M(p)$, at profile $p$ is essentially an approximate integral over the current downward trend up to $p$, moderated by a deceleration term based on the integral over the intervening upward trends. The deceleration gain parameter, $r$, defines how much weight to place on a step in the direction opposed to momentum. Algorithm 1 describes how downward momentum is calculated. Upward momentum, $U M(p)$, is treated independently and analogously. Note that momentum can never be negative.

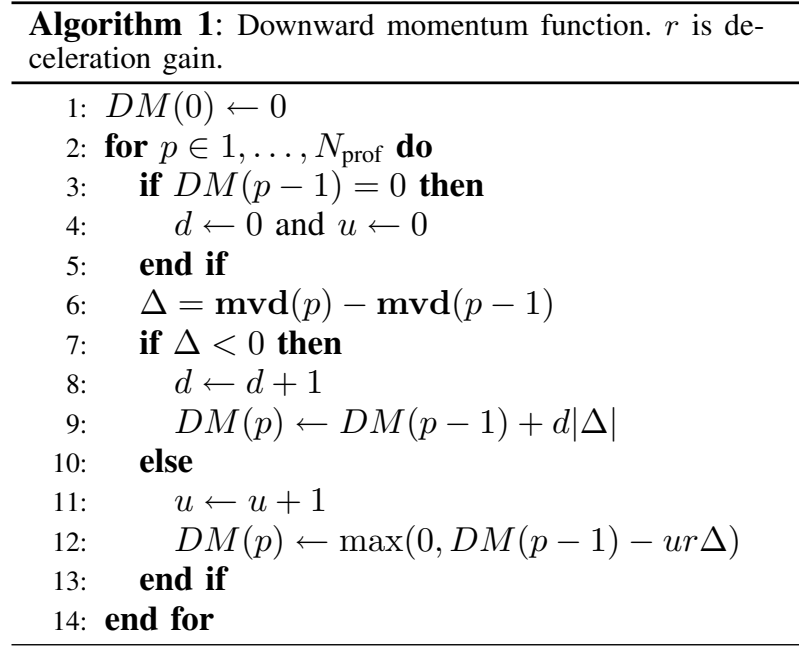

2) Determining the extent of fronts: Determining the actual extent of a front involves estimating both where the trend in mvd begins and ends. We do so in terms of the profile numbers at which momentum departs from zero and reaches a peak, respectively. Note that the zeros in the momentum function form a natural partition of the transect into regions where there is a likely trend and regions where there is not. We begin by defining index sets which contain potential fronts. Let $I_{1}, \ldots, I_{n w} \subset\left\{0, \ldots, N_{\text {prof }}\right\}, n w \in \mathbb{N}$, such that, for each $j \in\{1, \ldots, n w\}$

$$
\begin{gathered}
D M\left(\min \left(I_{j}\right)\right)=0, D M\left(I_{j} \backslash \min \left(I_{j}\right)\right) \neq 0 \\
\quad \text { and either } \\
\max \left(I_{j}\right)==N_{\text {prof }} \text {, or } D M\left(\max \left(I_{j}\right)+1\right)=0 .
\end{gathered}
$$

In other words, each $I_{j}$ defines a sequence of profiles which begin with zero momentum and carry through to the profile just before the momentum goes to zero again. We define $I_{\min }$ and $I_{\max }$ as:

$$
\begin{gathered}
I_{\min }=I_{\min }\left(I_{j}\right)=\underset{i \in I_{j}}{\operatorname{argmin}} D M(i) \\
I_{\max }=I_{\max }\left(I_{j}\right)=\underset{i \in I_{j}}{\operatorname{argmax}} D M(i),
\end{gathered}
$$

and note that by definition $I_{\min }\left(I_{j}\right)=\min \left(I_{j}\right)$, and $D M\left(I_{\min }\right)=0$. A first approximation of the extent of the potential front is the region $\left[I_{\min }, I_{\max }\right]$. 
The potential fronts are next filtered according to a relative threshold, $T_{\text {rel }} \in(0,1)$ (which ensures that this is a significant event within the dataset) and an absolute threshold $T_{\mathrm{abs}} \in \mathbb{R}$ (which allows for the possibility that no real front exists within the noisy data).

There are thus four control parameters used for tuning the MBFD based on historical data ( $T_{\text {rel }}, T_{\text {abs }}, r$, and the Kalman filter process noise). We used a tagged group of archived data sets with well-defined fronts to learn the best values for these parameters moving forward. For a given point in the parameter space, the front boundaries that were the results of MBFD were compared against the tagged boundaries and assigned a score based on a function of distance. The best values for our control parameters were the found using a simple hill-climbing algorithm to maximize the aggregate scores.

Each of the $I_{j}$ 's which is likely to contain a front can be refined further. This is because there may be a gradual change in momentum at the edges. In many cases, the region may be reduced considerably by taking into account the slope and curvature of the momentum. We do so here for one common case; the general treatment is the subject of ongoing work.

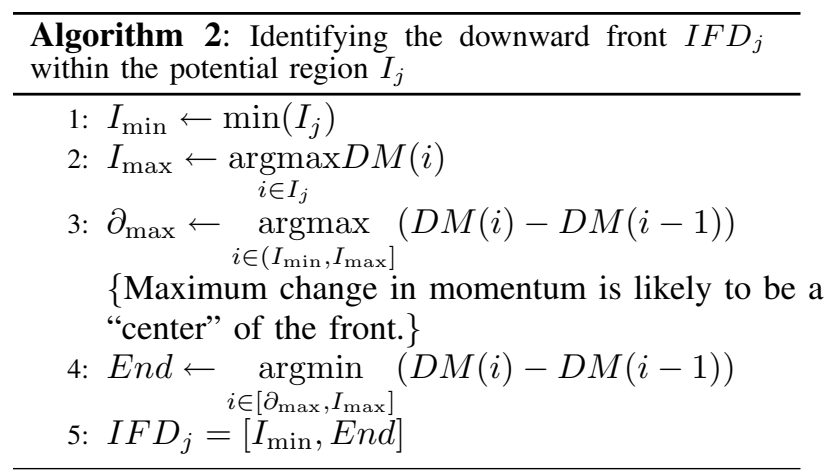

Analysis of past mission data shows that a sharp transition is often followed by a long plateau region as evident in Fig. 4. For this downward trend example, because the plateau is flat (with a very slight downward slope), the peak downward momentum value is in the middle of the plateau. It would be preferable for the end of the front to be identified with the point where the momentum first begins to flatten out. To this end, we identify the point at which the change in momentum is maximum, then look for the point of minimum change to the end of the window (Algorithm 2 describes this procedure in more detail). We will refer to the results of Algorithm 2 as the identified front (IFD for downward fronts, or $I F U$ for upward fronts).

\section{EXPERIMENTAL RESULTS}

\section{A. Trials on Archived Data}

MBFD was tested in two distinct ways. The first was an application to archived data. The second was executed online, during the course of a mission run in a CANON field experiment in September, 2011.
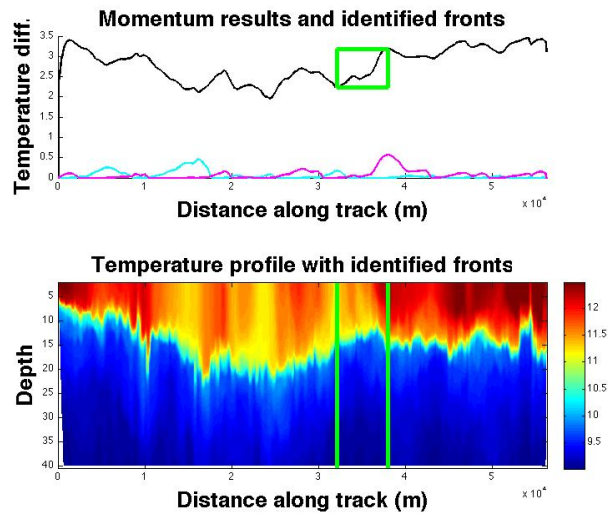

Fig. 5: Finding an ill-defined front from April, 2011, with mvd (black), DM (cyan), $U M$ (purple) and $I F U$ (green).

The 83 archived AUV data sets from 2009-2011 were categorized and partitioned into 3 groups based on the following heuristics used by oceanographers:

1) Well-defined fronts: These 17 data sets have clear fronts, as evidenced by a rapid temperature change in the horizontal direction. This is frequently, but not always, coincident with a large area of vertical homogeneity. Fig. 4 shows an example with the MBFD results superimposed.

2) No fronts: These 47 data sets clearly have no upwelling regions, and thus no frontal regions. Temperature is homogenous in the horizontal direction.

3) Ill-defined fronts: The remaining 19 data sets fall into this category. Fig. 5 shows an example along with the performance of MBFD. This example is atypical, as all but four of these sets are either characterized by corrupt data, or transitions between mixed and stratified water (see below).

We can also describe data sets in terms of the maximum depth the AUV attains in each profile. Stratified data sets are those where the maximum depth of every profile lies below the thermocline Mixed data sets are those where there are sustained periods where the maximum depth the AUV attains is above the thermocline, in the mixed layer, as well as periods where the maximum depth is below the thermocline. Fig. 6 gives an example.

Table I summarizes the performance results for MBFD on archived data sets with definitions for how results were classified. MBFD was successful at identifying well-defined fronts in stratified waters. Out of 14 such data sets, MBFD failed to identify the fronts in only 2. In two others it also generated false positive results, while still identifying the actual fronts. It was also reasonably successful at identifying data sets without fronts, correctly doing so in over half $(24 / 47)$ of the cases it was presented with. The vast majority $(83 \%)$ of the cases where MBFD returned a false positive for a data set with no fronts were the result of transitions between mixed and stratified waters. This is a consequence of using 
TABLE I: Performance of MBFD on archived AUV temperature data sets from 2009 to 2011 in Montery Bay, CA.

\begin{tabular}{|l|c|c|c|}
\hline Front Type & \multicolumn{2}{|c|}{ Well-defined } & \multicolumn{1}{|c|}{ None } \\
\hline Water Type & Stratified & Mixed & \\
\hline Number of Sets & 14 & 3 & 47 \\
\hline Successful sets & 10 & 1 & 24 \\
\hline Success percentage & 71 & 33 & 51 \\
\hline False positive sets & 2 & 0 & 20 \\
\hline Total FPs & 3 & 0 & 34 \\
\hline \hline $\begin{array}{l}\text { Successful set defi- } \\
\text { nition }\end{array}$ & $\begin{array}{l}\text { Identifies only } \\
\text { manually tagged } \\
\text { fronts }\end{array}$ & Finds no fronts \\
\hline $\begin{array}{l}\text { False Positive set } \\
\text { definition }\end{array}$ & $\begin{array}{l}\text { Finds all tagged } \\
\text { fronts plus other } \\
\text { results }\end{array}$ & $\begin{array}{l}\text { Only finds a front } \\
\text { due to moving } \\
\text { between mixed } \\
\text { and stratified } \\
\text { water }\end{array}$ \\
\hline
\end{tabular}

temperature difference in the water column, without taking into account average temperature or maximum depth of the water column.

While we've shown MBFD as applied to temperature, the algorithm can equally apply towards other parameters, such as salinity, nitrate or oxygen. Fig. 7 shows results for salinity and oxygen for the same June, 2011, mission.

\section{B. In-situ use of $\boldsymbol{M B F D}$}

MBFD was run simultaneously in three different contexts during a CANON field experiment conducted in Monterey Bay in September, 2011. During two overnight missions on September $7^{\text {th }}-8^{\text {th }}$, MBFD was run on-board the Dorado AUV, on shore accessing sub-sampled realtime data as it was sent back by the vehicle and postmission on the post-processed data set.

Weather conditions in the bay on these days were such that no upwelling was present to drive a strong front. From top to bottom, Fig. 8 shows first the in situ measurements indicating no front; then the results for on-shore sub-sampled data from the vehicle, which also indicates no front; and finally the temperature profile
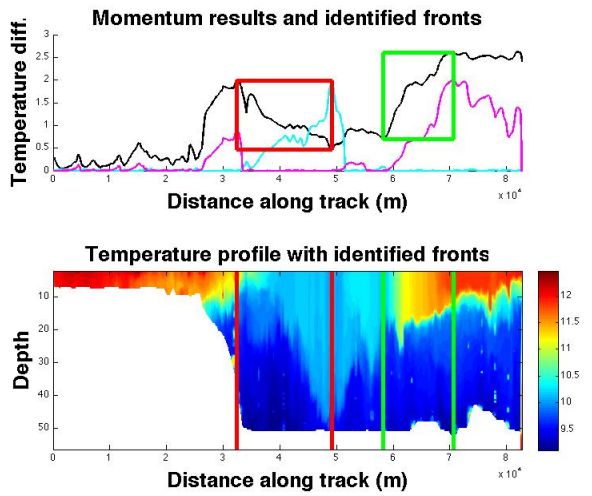

Fig. 6: Front-finding results for a mixed data set from March 25, 2009, with mvd (black), $D M$ (cyan), $U M$ (purple), IFD (red), and IFU (green).

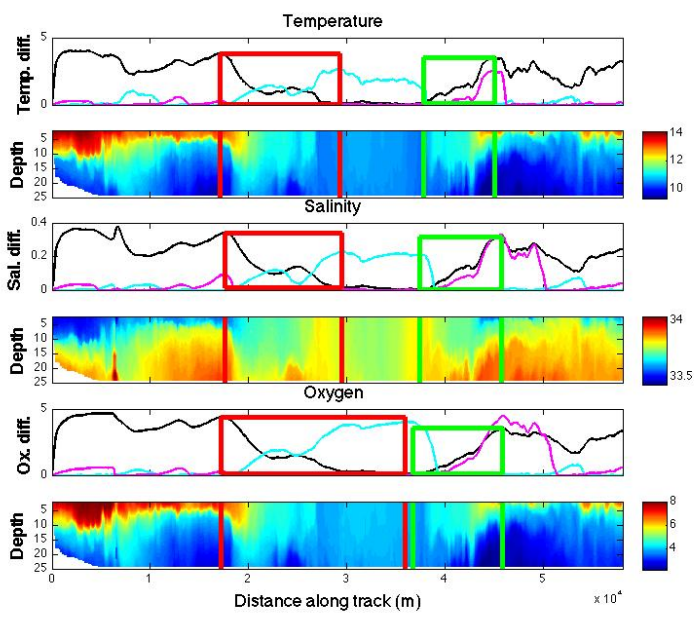

Fig. 7: Results of MBFD on post-processed temperature, salinity and $\mathrm{O}_{2}$ data for a mission June 2011. mvd (black), $D M$ (cyan), $U M$ (purple), IFD (red), and IFU (green).

for the post-processed data from the September $8^{\text {th }}$ run, where once again no front was detected. The general consensus of oceanographers during this experiment was that no strong front was visible. While not indicative of a strong confirmation for MBFD, the consensus of the results is generally encouraging.

\section{Discussion}

We show that MBFD does well in front detection. This is especially so when the AUV is driving exclusively through stratified waters. In this case, MBFD has been successful across a range of situated contexts, onboard and on-shore.

Two primary issues remain to be addressed. If the maximum depth of the AUV is below the thermocline, the temperature difference in a given water column will be large. If the AUV is driving in upwelled water, the temperature difference is going to be relatively low as upwelled waters are typified by being more homogenous than stratified waters. Due to strong correlations between water characteristics, this tends to be true for differences in other parameters as well. MBFD was designed to find the areas where these transitions occur.

However, if the maximum depth of the AUV is above the thermocline, as is the case with the mixed data sets, differences will be low there as well. This means MBFD will detect a downward trend in difference data both in the cases we want it to - when the AUV drives from stratified into upwelled water - and in spurious cases, such as when the maximum depth of the AUV shifts from below the thermocline to above the thermocline. This can be seen in the inaccurate downward front in Fig. 6. We are currently investigating how to differentiate these two cases.

The other challenge has to do with the geographic boundaries of the identified fronts, as reflected in Algorithm 2. The algorithm encapsulates heuristics often used 


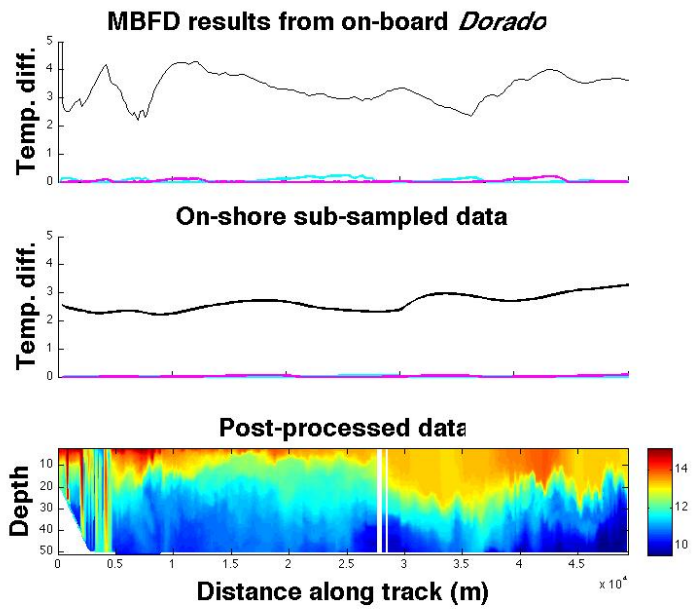

Fig. 8: Front finding results from September 7-8, 2011.

by oceanographers and therefore works well in cases where the front is sharply defined. However, MBFD has difficulty in disambiguating when there is a long, gently sloping gradient after the end of the front as shown in Fig. 7. The $I F D$ extends much farther into the upwelled region in the $\mathrm{O}_{2}$ plots than in the temperature or salinity plots because the $\mathrm{O}_{2}$ data shows a gently downward sloped region after the sharp transitions of the actual front have ended.

\section{Vi. CONClusions \& Future Work}

MBFD is a simple yet elegant algorithm for automatic front detection in a number of data-gathering contexts. It has been demonstrated on an AUV, on-shore while observing sub-sampled, real-time data sent back by the AUV and when examining high-resolution, postprocessed data.

A number of items are the focus for future work. A near-term goal is to use integrated multi-variate data at varying resolutions and frequencies on the AUV, to conclusively authenticate fronts onboard. Similarly, multiple data sources - remote sensing, multiple assets in CANON field programs and publicly available data such as [2] - would provide shore-side authentication. Combining estimates from all of these data sources into a near real-time estimate will be compelling.

Machine Learning provides another viable direction for automated parameter estimation in different contexts (e.g., in situ vs. on-shore); determining the appropriate means to mix results from different types of data and different data sources and to enable a more general purpose water-mass identifier to distinguish different characteristics.

Our long-term vision is to build an AUV controller which can enable multiple robots to autonomously explore the coastal ocean, respond to seasonal, dynamic and episodic phenomenon, collect data at high resolution and call home to report findings. MBFD is an important component in an early demonstration of this concept along with other ongoing work.

\section{ACKNOWLEDGMENTS}

MBARI authors are supported by the Lucille Packard Foundation. We thank our collaborators on CANON especially John Ryan and Jim Bellingham for their valuable support. This work was supported in part by a summer internship for Gottlieb at MBARI.

\section{REFERENCES}

[1] "CANON: Controlled, Agile and Novel Observing Network". [Online] Available: http://www.mbari.org/canon/.

[2] CENCoos. [Online] Available: http://www.cencoos.org/.

[3] Belkin, I. M., Cornillon, P. C., And Sherman, K. Fronts in large marine ecosystems. Progress In Oceanography 81, 1-4 (2009), 223 - 236. Comparative Marine Ecosystem Structure and Function: Descriptors and Characteristics.

[4] Cayula, J.-F., And Cornillon, P. Multi-Image Edge Detection for SST Images. Journal of Atmospheric and Oceanic Technology 12, 4 (2011/09/13 1995), 821-829.

[5] CRUZ, N., AND Matos, A. Adaptive sampling of thermoclines with autonomous underwater vehicles. In Proc. OCEANS 2010 (September 2010), pp. 1-6.

[6] Das, J., Maughan, T., McCann, M., Godin, M., O'Reilly, T., Messié, M., Bahr, F., Gomes, K., Py, F., Bellingham, J., Sukhatme, G. S., AND RaJan, K. Towards Mixedinitiative, Multi-robot Field Experiments: Design, Deployment and Lessons Learned. In Proc. IROS (2011).

[7] D'Asaro, E., Lee, C., Rainville, L., Harcourt, R., And Thomas, L. Enhanced turbulence and energy dissipation at ocean fronts. Science 10 (March 2011).

[8] Haney, J., AND McGillivary, P. Midshelf fronts in the south atlantic bight and their influence on seabird distribution and seasonal abundance. Bio. Oceanography 3 (1985), 401-430.

[9] Hopkins, J., Challenor, P., And Shaw, A. G. P. A new Statistical Modeling approach to ocean front detection from SST satellite images. JTech 27 (2010), 173-191.

[10] Longhurst, A. Ecological Geography of the Sea. Academic Press, 2006

[11] Marcello, J., Marques, F., and Eugenio, F. Automatic tool for the precise detection of upwelling and filaments in remote sensing imagery. Geoscience and Remote Sensing, IEEE Transactions on 43, 7 (july 2005), 1605-1616.

[12] Olaya, A., Py, F., Das, J., And Rajan, K. An On-line Utility based Approach for Sampling Dynamic Ocean Fields. IEEE Journal of Ocean Engineering (2011). Accepted for publication.

[13] Olson, D., Hitchcock, G., Mariano, A., Ashuan, C., Peng, G., Nero, R., And Podesta, G. Life on the edge: marine life and fronts. Oceanography 7 (1994), 52-60.

[14] ORam, J. J., MCWilliams, J. C., ANd StolzenbaCh, K. D. Gradient-based edge detection and feature classification of seasurface images of the southern california bight. Remote Sensing of Environment 112, 5 (2008), 2397 - 2415. Earth Observations for Terrestrial Biodiversity and Ecosystems Special Issue.

[15] Pingree, R. D., And Griffiths, D. K. Tidal fronts on the shelf seas around the british isles. JGR 83 (1978), 4615-4622.

[16] Py, F., Ryan, J., T.O.Reilly, Thomas, H., and Rajan, K. Following Ocean Fronts by Dynamically Retargeting AUVs from Shore. Mediterranean Marine Sci. J, Special Issue on AUVs (2010). Submitted, under review.

[17] Zhang, Y., Godin, M., Bellingham, J. G., and Ryan, J. P. Ocean Front Detection and Tracking by an Autonomous Underwater Vehicle. In Proc. MTS/IEEE OCEANS (September 2011), pp. 1-4.

[18] Zhang, Y., Ryan, J. P., Bellingham, J. G., Harvey, J., McEwen, R., Chavez, F., And Scholin, C. Classification of Water Masses and Targeted Sampling of Ocean Plankton Populations by an Autonomous Underwater Vehicle. In $A G U$ Fall Meeting Abstracts OS21A-1609 (December 2011). 